\title{
Antecedentes do Comprometimento Calculativo de Clientes de TI Pós Falha e Recuperação de Serviços em Contextos B2B
}

\section{Antecedents of Calculative Commitment of TI Clients Post-Failure and Recovery of Services in B2B Contexts}

\author{
Humberto Caetano Cardoso da Silva ${ }^{1}$, Marcus Augusto Vasconcelos Araújo ${ }^{2}$ \\ ${ }^{1}$ Universidade Federal de Pernambuco, UFPE, Brasil \\ ${ }^{2}$ Faculdade Boa Viagem, FBV, DeVry, Brasil \\ Correspondência: Humberto Caetano Cardoso da Silva, Endereço: Av. Santos Dumont, 1011, sl 107, Rosarinho, \\ CEP: 52041060, Recife, PE, Brasil. Tel.: 5581 3212-1224. E-mail: humberto.ccs@gmail.com
}

Recebido: 23 de dezembro de 2016 Aceito: 27 de agosto de 2017 Publicado: 31 de outubro de 2017

DOI: http://dx.doi.org/10.21714/1679-18272016v14n2.p343-354

\begin{abstract}
Resumo
O ideal seria que falhas de serviço não existissem, mas os erros e falhas são inerentes do processo de prestação de serviços. A severidade da falha, percebida em um ambiente de grande competitividade como é o ambiente B2B, pode levar ao fim do relacionamento cliente-fornecedor. A qualidade da recuperação e os resultados que estas recuperações geram, podem criar índices de confiança e comprometimento entre o cliente e o fornecedor que irão reforçar os laços comerciais, gerando relacionamentos duradouros. A dependência do cliente pode ser um dos motivos pelos quais estes se mantêm em relações, mesmo quando insatisfeitos com a prestação do serviço. Este trabalho estudou a relação entre o comprometimento calculativo e a confiança, obtidos após uma recuperação de falha de serviço, juntamente com a severidade percebida da falha de serviços e a dependência do fornecedor, em ambientes bussiness-to-bussiness (B2B) após a ocorrência de uma falha de serviços. O estudo foi realizado com profissionais de TI que ocupam cargo de chefia e, portanto, exercerem o poder de encerrar relacionamentos com fornecedores. A análise dos dados foi realizada a partir da utilização da técnica de MEE, buscando, dessa maneira, verificar as relações das variáveis em um modelo único.
\end{abstract}

Palavras-chave: Falhas de Serviços, B2B, Comprometimento Calculativo, Confiança.

\begin{abstract}
The ideally would be that service failures do not exist, but errors and failures are inherent in the service delivery process. The severity of the failure, perceived in a highly competitive environment such as the B2B environment, can lead to the end of the customer-supplier relationship. The quality of the recovery and the results that these recoveries generate can create confidence levels and compromise between the customer and the supplier that will strengthen the commercial ties, generating lasting relationships. The client dependence on the supplier may be one of the reasons why they remain in relationships, even when dissatisfied with the provision of the service. This work studied the relationship between the calculative commitment and the trust obtained after a service failure recovery, together with the perceived severity of service failure and supplier dependency in Bussiness-toBusiness (B2B) environments after the occurrence of a service failure. The study was carried out with IT professionals who hold leadership positions and, therefore, exercise the power to terminate relationships with suppliers. The data analysis was performed using the SEM technique, to verify the relations of the variables in a single model.
\end{abstract}

Keywords: Service Failure, B2B, Calculative Commitment, Trust.

Esta obra está licenciada sob uma Licença Creative Commons Attribution 3.0.

\section{Introdução}

O processo de terceirização de Tecnologia da Informação (TI) tem gerado novas oportunidades de se empreender em ambientes Business-to-Business (B2B). Esta terceirização surge da necessidade da redução de custos e pessoal objetivando que as organizações consigam atingir seus objetivos, com a qualidade e o desempenho necessários do TI (BERNSTORFF; CUNHA, 2000). 
http://www.revista.ufpe.br/gestaoorg

Existe um reconhecimento geral de que os clientes, assim como os produtos, têm um ciclo de vida que os acompanha durante a sua relação com as empresas. Inicialmente os clientes são prospectos, em seguida clientes novatos, clientes costumeiros e, finalmente, defensores da marca (ANG; BUTTLE, 2006).

Em relacionamentos de longo prazo falhas na prestação de serviços podem ocorrer e prover "erro zero" deve ser o objetivo de qualquer organização, mas, em se tratando de serviços, devido à sua natureza variável, entregas e resultados são diferentes ao longo do tempo (LOVELOCK et al., 2011). Estes “diferentes resultados" podem ser encarados pelos clientes como falhas e as mesmas tendem a ser ubíquas na indústria de serviços (BERRY; PARASURAMAN, 1991) e mais perceptíveis em ambientes de alta competitividade como ambientes B2B.

Uma boa recuperação da falha é um fator de construção de relações de longo prazo com clientes que, inicialmente, encontravam-se insatisfeitos (TAX et al., 1998; WEUN; BEATTY; JONES, 2004). Tax et al. (1998) argumentam que uma recuperação de serviços de alta qualidade pode gerar maior percepção de comprometimento e confiança do cliente no fornecedor, sendo esses dois construtos, comprometimento e confiança, os primeiros a serem testados no modelo teórico.

A falha em ambientes altamente competitivos, como o ambiente de relações B2B, pode gerar altos níveis de insatisfação, e quanto maior a severidade dessa falha, maior será a necessidade de uma recuperação de serviço de qualidade. Em casos extremos, não importa o quão bem realizada foi a recuperação do serviço, pois o cliente percebe uma falha tão grave que qualquer compensação que a empresa forneça não será suficiente (McCOLLOUGH, 2009).

A partir do momento da falha e da consequente recuperação de serviços, o compromisso entre as partes, proveniente de uma alta qualidade na recuperação, é a crença de que um relacionamento vale a pena ser mantido, e que se possam justificar esforços para que este relacionamento tenha longa duração (MORGAN; HUNT, 1994). Já a confiança é a aceitação de riscos pelas partes envolvidas em um relacionamento, entendendo que existem aspectos básicos de honestidade, boa vontade e competência (SHEPPARD; SHERMAN, 1998). Gounaris (2005) argumenta que comprometimento e confiança são duas noções altamente inter-relacionadas, que estimulam um vínculo emocional entre o fornecedor e o cliente, que facilita o estabelecimento de relações colaborativas, reduz incertezas e aumenta a eficiência na utilização de recursos.

Wetzels et al. (1998) argumentam que existem dois tipos de comprometimento o calculativo e o afetivo. O comprometimento calculativo pode ser compreendido como uma avaliação cognitiva do valor da continuação de um relacionamento. O comprometimento calculativo é, portanto, um estado de ligação à um fornecedor, pois o final dessa relação implicaria sacrifícios e perdas conquistadas ao longo do tempo. Um aspecto importante do comprometimento calculativo é o reconhecimento de altos custos de mudança associados ao fim do relacionamento (SIQUEIRA, 2001).

Já a dependência é definida como a ausência de alternativas melhores ou equivalentes disponíveis no mercado (GAO et al., 2005), sendo um antecedente importante para o comprometimento e a confiança na relação (GEYSKENS et al., 1996; VENETIS; GHAURI, 2004). Segundo Gilliland e Bello (2002), uma relação de dependência se estabelece quando uma das partes tem mais a perder com o fím da relação que a outra parte, pois a parte dependente terá dificuldades em obter os níveis de serviço e benefícios existentes na relação atual.

A presente pesquisa foi desenvolvida com a intenção de testar um modelo teórico a partir da utilização da técnica de Modelagem de Equações Estruturais (MEE), no sentido de investigar a relação entre os antecedentes do comprometimento calculativo de clientes em ambientes B2B no setor de Tecnologia da Informação em situações pós-falha e aplicação de ação de recuperação de serviços, de forma a verificar seu poder explicativo.

Neste cenário, apresenta-se a questão de pesquisa que norteou este trabalho: Qual a relação entre o comprometimento calculativo de clientes com a severidade da falha, a confiança e a dependência do fornecedor após a ocorrência de falhas de serviços de TI em contextos B2B?

Este trabalho está estruturado em cinco sessões. Inicialmente serão apresentados o mercado de TI e o mercado B2B, assim como falha e recuperação de serviços. A segunda parte apresenta os construtos abordados no estudo, e o modelo teórico proposto. Na terceira parte serão apresentados os procedimentos metodológicos. A quarta seção trará os resultados obtidos a partir da coleta de dados. E, finalmente, na quinta parte, serão apresentadas as considerações finais do estudo.

\section{Fundamentação Teórica}

Nesta seção será iniciada a discussão teórica sobre o ambiente de Tecnologia da Informação nas relações B2B, dos serviços de suporte e recuperação de falhas, dos construtos estudados e sua relação com a retenção de clientes.

\subsection{O Serviço de Tecnologia da Informação e o Ambiente B2B}


A característica de ser cliente e provedor de serviços ao mesmo tempo, faz com que o setor de TI das empresas desempenhe, também, o papel do tomador de serviços. Essas relações desenvolvidas entre empresas têm aspectos especiais, pois se desenvolvem não só do ponto de vista de ser uma prestação de serviços em um do tipo mercado B2B (Business-to-Business), mas também por serem estes serviços, em grande parte, de alta intangibilidade, visando a entrega de um serviço final, que também é intangível (LOVELOCK, 2011).

$\mathrm{O}$ aspecto de alta intangibilidade dos serviços de TI, e o tipo entrega de serviço que é realizado, quase sempre através de ferramentas self-service, trazem outros desafios para a prestação deste tipo de serviço. Desafios estes já tratados em outros estudos (BITNER, 1990; BITNER, 2001; CURRAN et al. 2003), que pesquisaram a entrega de serviços através da utilização de tecnologias self-service. Esta tecnologia self-service, inerente a entrega do serviço de TI, traz várias dificuldades para que sejam alcançados altos níveis de satisfação do cliente. (BEATSON et al., 2007).

Adicionalmente, a TI proporciona a capacidade de obtenção de vantagens competitivas ao longo da "cadeia de valor" (PORTER; MILLAR, 1985). O conjunto de cada uma das atividades tecnológicas, utilizadas pela empresa, na realização dos seus negócios é a chamada "cadeia de valor". A TI pode gerar barreiras à entrada de novos competidores, como nos casos em que apenas uma empresa tem certa tecnologia ou ferramenta e oferece um serviço diferenciado, influenciar a negociação com fornecedores, como nos casos de ferramentas se supply chain, alterar a base de competição e, até mesmo, gerar novos produtos, que só podem ser oferecidos por conta da utilização de TI (MCFARLAN, 1984; ALBERTIN; ALBERTIN, 2012).

\subsection{Serviço e Falha de Serviço}

Utilizando a classificação de duração da prestação de serviços, proposta por Hume e McColl-Kennedy (1999), excetuando-se os casos de manutenção física de equipamentos, que podem ser episódicos ou contínuos, a prestação de serviços de Tecnologia da Informação a empresas é dada de maneira contínua. Neste contexto, e do ponto de vista da "flor de serviços" proposta por Lovelock (1994), o chamado "suporte à Tecnologia da Informação" é a recuperação de serviço a ser realizada no momento de uma falha ou redução da qualidade na prestação de um serviço de tecnologia.

A satisfação ou insatisfação do cliente durante a utilização de um serviço podem ser observadas sob a ótica do paradigma da desconfirmação de expectativas (CHURCHILL; SURPRENANT, 1982; OLIVER, 1980; OLIVER; DESARBO, 1988; TSE; WILTON, 1988; SILVA; ARAÚJO, 2015). Nele o processo cognitivo que o cliente utiliza para concluir que a prestação de serviços foi satisfatória, passa pela avaliação da expectativa existente antes do encontro de serviços. Caso a performance ou resultado observados durante o encontro de serviços tenha sido igual, ou mesmo maior, que a expectativa anterior, o encontro foi satisfatório. Caso contrário, a performance ou o resultado observado durante o encontro de serviços tenha sido inferior a expectativa anterior o encontro foi insatisfatório, gerando a falha de serviços (BITNER, 1990; SILVA; ARAÚJO, 2015).

Recuperação de serviço são todas as atividades associadas à intenção ou esforços que a empresa realiza no sentido de identificar e corrigir as eventuais falhas de serviço (TAX et al., 1998; WANG; CHANG, 2013). Como falhas são inevitáveis, é necessário que se criem procedimentos e controles de forma a identificar quão frequentes são estas falhas e como se recuperar após estas ocorrerem (WANG et al., 2011). Tax et al. (1998) propõem que satisfação com o processo de recuperação irá afetar positivamente o comprometimento e a confiança do cliente, resultando em situações nas quais a satisfação do cliente com o provedor de serviços é maior pós a falha que antes da falha (TAX et al., 1998; WANG et al., 2011; WANG; CHANG, 2013).

Em ambientes B2B, independentemente do tamanho do investimento feito pelo cliente na relação, o término de uma relação sempre será seguido por uma nova relação, podendo esta ser estabelecida com provedores de serviço externos ou internos. Outros fatores além das relações pessoais influenciam o comportamento de retenção em ambientes B2B, como custos de mudança, qualidade na recuperação do serviço e inércia do cliente (WHITE; YANAMANDRAM, 2007; YANAMANDRAM; WHITE, 2010).

\subsection{Severidade da Falha}

Devido à natureza variável do serviço as falhas são inevitáveis. A falha de serviço pode ocorrer quando o serviço não atende às expectativas do cliente. Vários estudos argumentam que a severidade da falha deve ser levada em consideração quando está sendo analisada a falha e recuperação de serviço. A severidade da falha pode ser definida como a severidade percebida pelo cliente do problema, quanto mais intensa a severidade de um problema, maior será a perda percebida pelo cliente (WANG et al., 2011).

Weun et al. (2004) argumentam que, apesar de vários estudos tratarem de falha e recuperação de serviços, estes não incluem a severidade da falha nos seus modelos, apesar de autores identificarem que a severidade da falha é importante fator a ser considerado no processo de recuperação de serviços. Os autores adicionam que a severidade da falha terá influência na avaliação de um provedor de serviços após a falha ter sido recuperada, e que, apesar de uma recuperação de serviços eficiente, uma falha com alto grau de severidade produziria uma perda perceptível para o cliente (WEUN et al., 2004). 
http://www.revista.ufpe.br/gestaoorg

Ainda segundo Weun et al. (2004), uma falha de serviço com um alto grau de severidade percebida pelo cliente tende a ter uma relação negativa com a satisfação, a confiança e o comprometimento, e uma relação positiva com o boca-a-boca negativo. Os resultados encontrados pelos autores deram suporte para as quatro hipóteses. Concomitantemente Wang e Chang (2013) argumentam que a severidade da falha percebida pelo cliente é um fator crítico que influencia na qualidade do relacionamento, isto é, na confiança e no comprometimento. Finalmente, McQuilken (2010) acrescenta que a confiança percebida pelo consumidor terá um impacto negativo caso este se depare com uma situação de falha de serviço com alto grau de severidade.

\subsection{Dependência do Fornecedor}

Gao et al. (2005) define dependência como a ausência de alternativas melhores ou equivalentes disponíveis no mercado. Venetis e Ghauri (2004) acrescentam que a dependência de uma parte em relação à outra é um importante antecedente para o comprometimento na relação. Segundo Gilliland e Bello (2002), uma relação de dependência se estabelece quando uma das partes tem mais a perder com o fim da relação que a outra parte, pois a parte dependente terá dificuldades em obter os níveis de serviço e benefícios existentes na relação atual.

O modelo de investimento em relações pessoais amplia as proposições da teoria de interdependência de forma a analisar a tendência a se manter em uma relação. A dependência é uma figura central na estrutura da interdependência, na busca do entendimento da persistência em um relacionamento. O "Nível de dependência" se refere à extensão da necessidade de um indivíduo se manter em uma relação (RUSBULT et al.,1998; YANAMANDRAM; WHITE, 2010).

Yanamandram e White (2010), White e Yanamandram (2007) e Chang et al. (2012), são alguns dos autores que avaliaram o papel da dependência do fornecedor em ambientes B2B e sua relação com retenção, comprometimento afetivo e comprometimento calculativo. Yanamandram e White (2010) argumentam que em um contexto B2B, as relações entre comprador-fornecedor, também envolvem relações e interações pessoais. Estes laços sociais servem para aumentar a dependência do cliente no fornecedor de serviços. Os autores acrescentam, ainda, que a inabilidade do cliente em substituir o fornecedor também pode ser considerada uma indicação do grau de dependência que um cliente tem do seu fornecedor.

Em ambientes de relações B2B, o investimento no aprimoramento da relação cliente-fornecedor é importante pois ele não só aumenta os custos de mudança, como também torna a relação mais próxima, criando sinergia entre as partes. Quando os clientes percebem que os fornecedores estão realizando um investimento na relação, tentando torna-la mais forte, sua dependência do fornecedor irá aumentar (CHANG et al., 2012).

A dependência é um processo de manutenção da relação dinâmico, sendo determinado por ambas as partes pelos recursos e pelo poder na relação. Clientes com menor poder na relação ou menor quantidade de recursos, tem maior dependência do fornecedor e, por conseguinte, enfrenta situações de maior perda no momento de encerrar um relacionamento. Em situações de alta dependência o cliente se vê forçado a calcular os sacrifícios e perdas do encerramento de uma relação. Dessa forma a dependência é positivamente relacionada com o comprometimento calculativo (GEYSKENS et al., 1996; GILLILAND; BELLO, 2002; CHANG et al., 2012; YANAMANDRAM; WHITE, 2010).

\subsection{Confiança}

A confiança é um dos construtos mais importantes em contextos relacionais, sendo de grande importância no desenvolvimento de relações cliente-fornecedor de longo prazo (MORGAN; HUNT, 1994; MOUZAS et al., 2007; JIANG et al., 2011). Palmatier et al. (2013) declaram que "É esperado que a confiança afete a velocidade com a qual ocorre o comprometimento de forma positiva. Confiança é um aspecto importante na relação, pois cria um ambiente no qual o aprendizado e a adaptação podem ocorrer”.

Confiança é um aspecto central do marketing de relacionamento, e assume papel de grande importância em ambientes de serviço B2B, na medida em que os compradores se deparam com grande complexidade na avaliação de muitos aspectos intangíveis do serviço oferecido. Agregado à incerteza da capacidade de o fornecedor atender às expectativas do cliente de forma consistente, os clientes devem considerar aspectos da relação que sugerem que o fornecedor está alinhado às necessidades do cliente (DONEY et al. 2007).

Tax et al. (1998) e Gounaris (2005) propõem que quanto maior a qualidade percebida no serviço, ou na recuperação do mesmo, maior será a o grau de confiança que o cliente terá no fornecedor. Corroborando com esta afirmação, Doney et al. (2007) afirmam que os aspectos de qualidade técnica e funcional do serviço estão relacionados positivamente com a confiança do cliente no provedor de serviços.

A confiança em uma pessoa ou organização se desenvolve através da observação ou do aprendizado de sucessivas interações. Em situações de falhas de serviço, o comportamento, as ações e o histórico delas contribuem para a criação da relação de confiança entre cliente e fornecedor. Como a satisfação está ligada diretamente à confirmação das expectativas, a satisfação com recuperações de serviço contribui para a 
confiabilidade da empresa e, assim, para a relação de confiança (TAX et al., 1998; PIZZUTTI DOS SANTOS; BASSO, 2012; WANG; CHANG, 2013).

\subsection{Comprometimento Calculativo}

Segundo Morgan e Hunt (1994), o marketing de relacionamento se refere a todas as atividades direcionadas a estabelecer, desenvolver e manter relações de sucesso. Morgan e Hunt (1994) definem compromisso como uma relação contínua tão importante que o "parceiro de troca", ou a "outra parte na relação", possa justificar o esforço máximo para manter essa relação. As partes do compromisso acreditam que o relacionamento vale a pena ser mantido e se asseguram que esta relação dure longo tempo. Os autores também propõem que o comprometimento na relação é um dos fatores centrais do marketing de relacionamento.

Relacionamentos baseados no comprometimento calculativo, no momento de encerramento dos mesmos, passam por análises de custo/benefício, sendo os custos associados ao fim do relacionamento e os benefícios esperados no novo relacionamento (WHITE; YANAMANDRAM, 2007; YANAMANDRAM; WHITE, 2010).

Um ponto identificado na literatura é a diferença entre dependência do cliente em relação ao fornecedor e comprometimento calculativo. A dependência está ligada a elementos estruturais que mantém a relação, enquanto que o comprometimento calculativo se relaciona ao grau de motivação que existe na continuação de uma relação baseada em laços estruturais (GEYSKENS et al., 1996). Apesar de semelhantes, os dois construtos, dependência e comprometimento calculativo, não são sinônimos, e alguns estudos tratam o construto dependência como um antecedente do comprometimento calculativo (HEIDE; JOHN, 1992; VENETIS; GHAURI, 2004).

Chang et al. (2012) investigam como o comprometimento, tanto o calculativo como o afetivo, em ambientes de relações B2B, são alcançados a partir de fatores como custos de término do relacionamento, dependência do cliente em relação ao fornecedor, custos de perda de investimentos feitos no relacionamento cliente e fornecedor e confiança. Ao final do estudo os autores demonstram existir uma relação entre os construtos.

\subsection{Hipóteses do Estudo}

A partir dos construtos identificados no presente estudo como antecedentes do comprometimento calculativo, é possível serem identificadas hipóteses e relações teóricas a serem avaliadas de maneira empírica.

Os construtos comprometimento calculativo e confiança, têm sido relacionados em alguns estudos como de Wang e Chang (2013), que tratam de situações de recuperação de falhas de serviços em restaurantes, identificaram uma relação entre a confiança e o comprometimento. Gounaris (2005) e Chang et al. (2012) também corroboram com essa possível relação, porém Chang et al. (2012) propõem que a relação da confiança com o comprometimento calculativo é negativa. Com base nisso, percebe-se a oportunidade de se verificar empiricamente a confiança e a sua relação com o comprometimento calculativo do cliente em relação ao fornecedor e, sendo assim, apresenta-se a hipótese de pesquisa $\mathbf{H 1}$ :

H1: $O$ comprometimento calculativo de clientes é positivamente relacionado à confiança percebida quando a recuperação de falhas de serviço é considerada satisfatória pelos clientes.

Para a variável dependência, Geyskens et al., (1996) argumentam que, em uma relação de dependência, a não exploração dessa dependência pela parte mais forte da relação pode gerar maiores níveis de confiança no fornecedor. Beldad et al. (2010), a partir de uma revisão literária, teorizam que, em relações de dependência, o cliente passa a confiar que o fornecedor irá cumprir com o que promete, aumentando os níveis de confiança por parte do cliente. Sendo assim, apresenta-se a hipótese de pesquisa $\mathbf{H 2}$ :

H2: A confiança percebida pelo cliente no fornecedor é positivamente relacionada à dependência do cliente em relação ao fornecedor.

Adicionalmente, Geyskens et al. (1996), Gilliland e Bello (2002), Gounaris (2005), Chang et al. (2012) e Yanamandram e White (2010), propuseram que a dependência do fornecedor tem relação com o comprometimento. Dessa forma é possível apresentar a hipótese de pesquisa H3:

H3: $O$ comprometimento calculativo do cliente em relação ao fornecedor é positivamente relacionada à dependência do cliente em relação ao fornecedor.

Finalmente, Weun et al. (2004) argumentam que a severidade da falha tem um impacto negativo na confiança, no comprometimento e no boca-a-boca positivo. Concomitantemente, Wang e Chang (2013) afirmam que a severidade da falha percebida pelo cliente é um fator crítico da qualidade do relacionamento, isto é, na confiança e no comprometimento. Dessa forma, apresentam-se as hipóteses de pesquisa H4 e H5.

H4: A severidade da falha de serviços é negativamente relacionada à confiança do cliente no fornecedor.

H5: A severidade da falha de serviços é negativamente relacionada ao comprometimento calculativo do cliente no fornecedor. 
O modelo teórico proposto no presente estudo, apresentado na Figura 1, foi desenvolvido a partir dos modelos teóricos propostos por Tax et al. (1998), Weun et al. (2004), White e Yanamandram (2007), Yanamandram e White (2010) e Wang et al. (2011).

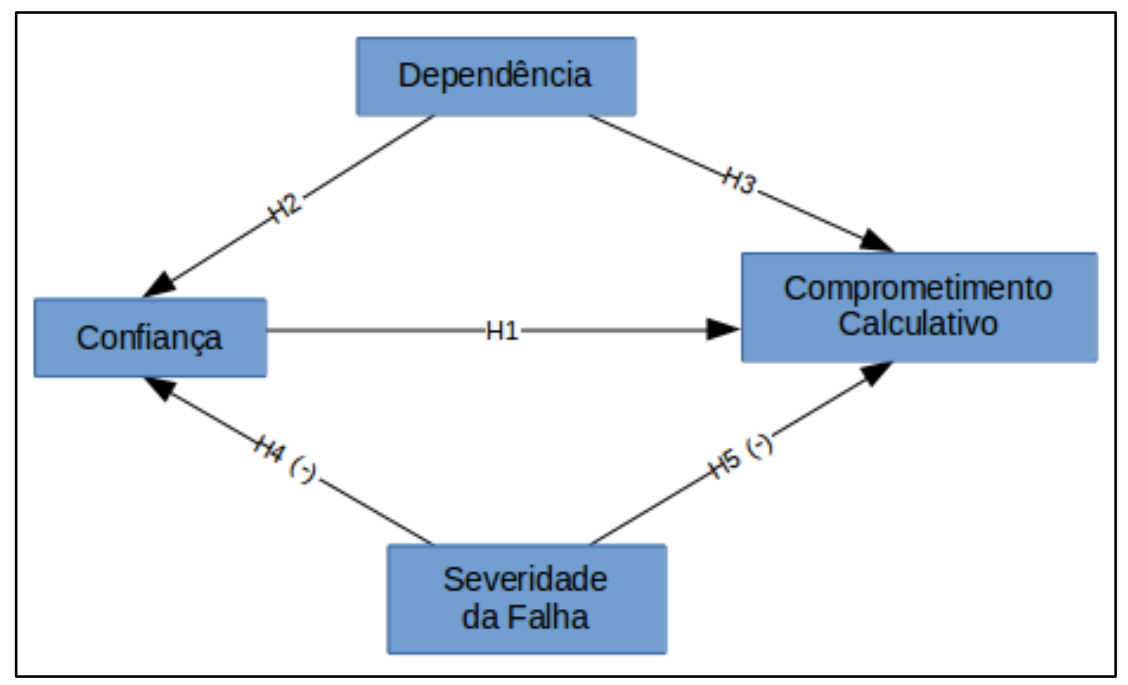

Figura 1: Modelo Teórico Proposto.

Fonte: Elaborado pelos autores.

\section{Procedimentos Metodológicos}

Esta pesquisa utilizou os pressupostos sugeridos por Hair Jr., Black, Babin, Anderson e Tatham (2009) e Malhotra, Birks e Wills (2012) para estudos de natureza quantitativa, caracterizando esta pesquisa como descritiva, com a utilização de um levantamento (survey), com corte transversal único.

De acordo com o modelo teórico apresentado na Figura 1, quatro construtos foram abordados e testados. A Tabela 1 apresenta as variáveis que foram pesquisadas através da survey e os trabalhos que serviram como referência para a criação do instrumento de pesquisa.

Tabela 1. Escalas Utilizadas no Estudo.

\begin{tabular}{|c|c|}
\hline Construtos & Estalas Utilizadas \\
\hline $\begin{array}{l}\text { Confiança } \\
\text { - Este fornecedor cumpre as promessas feitas. } \\
\text { - Este fornecedor sempre é honesto comigo. } \\
\text { - Eu acredito nas informações que este fornecedor me fornece. } \\
\text { - Este fornecedor está verdadeiramente preocupado comigo. } \\
\text { - } \text { Quando decisões importantes são tomadas, este fornecedor considera o } \\
\text { - } \text { Eu confio que este fornecedor considere nossos melhores interesses. } \\
\text { - Este fornecedor é confiável. }\end{array}$ & $\begin{array}{l}\text { Doney e Cannon } \\
\text { (1997); De } \\
\text { Andrade et al. } \\
\text { (2011); Nespolo } \\
(2014)\end{array}$ \\
\hline $\begin{array}{l}\text { Dependência do Fornecedor } \\
\text { - Este fornecedor é crucial para nossa performance futura. } \\
\text { - Nós somos dependentes desse fornecedor. } \\
\text { - Este fornecedor é importante para nosso negócio. }\end{array}$ & $\begin{array}{l}\text { Ganesan (1994); } \\
\text { Yanamandram e } \\
\text { White (2010); } \\
\text { Chang et al. (2012) }\end{array}$ \\
\hline $\begin{array}{l}\text { Severidade da Falha } \\
\text { • Este problema afetou gravemente o meu negócio. }\end{array}$ & $\begin{array}{l}\text { Weun et al. (2004); } \\
\text { Tang }\end{array}$ \\
\hline
\end{tabular}


- Este problema prejudicou o meu negócio.

(2014)

- Este problema causou uma grande perda para o meu negócio.

Comprometimento Calculativo

- Vale a pena economicamente ser um cliente desse fornecedor.

- A empresa iria sofrer economicamente se o relacionamento fosse al., (2012) quebrado.

- O fornecedor tem vantagens de localização em comparação com outros fornecedores.

\section{Fonte: Elaborado pelos autores}

Considerando a técnica de informantes-chave, que propõe que os informantes devem ser selecionados com base nas suas qualificações, no conhecimento especializado e na sua posição na empresa, a pesquisa será realizada em empresas de setores variados da economia do Brasil. Os participantes do estudo foram gestores de Tecnologia da Informação. Este cargo foi escolhido, pois são estes que recebem o serviço de suporte, que contratam as empresas terceirizadas e que têm que reportar à alta administração os sucessos e as falhas do serviço (KUMAR; STERN; ANDERSON, 1993).

A forma de coleta de dados definida para esta pesquisa se deu a partir de duas maneiras: (1) o questionário foi entregue pelo próprio pesquisador em empresas nas quais o mesmo tem acesso à equipe decisora em questões relacionadas a TI, e (2) através da utilização de alunos de graduação em cursos de Sistemas de Informação, Ciência da Computação e Redes de Computadores, que atuaram como pesquisadores junto aos gestores das empresas às quais estão trabalhando. Essa metodologia já foi utilizada em outros estudos como os de Bitner, Booms e Tetreault (1990) e Jones et al. (2007). De maneira a confirmar a consistência dos questionários entregues, das 134 empresas participantes do estudo, 30 foram escolhidas aleatoriamente e contatadas, não sendo encontrado nenhuma inconsistência.

Em relação à técnica de análise das relações entre as variáveis do estudo, foi utilizada a modelagem de Equações estruturais (MEE), também conhecida pela sigla SEM (do inglês Structural Equation Modeling). Segundo Hair Jr. et al. (2009), a utilização desta abordagem de MEE é benéfica pois melhora a estimação estatística, demonstra uma melhor representação dos conceitos teóricos e explica diretamente o erro de mensuração, ou seja, ele pode assumir erros atribuídos à falta de um modelo de ajuste. Considerando que o objetivo central deste trabalho é verificar as relações de construtos a partir da proposição de um modelo teórico, a utilização da MEE possibilita a análise do conjunto de construtos propostos em um modelo de interações único.

De acordo com Hair Jr. et al. (2009), "A validade de um modelo de mensuração depende da qualidade de ajuste para o mesmo e evidência específica de validade de construto". A qualidade do ajuste (GOF - Goodness-of-Fit), indica se o modelo especificado reproduz a matriz de covariância entre os itens indicadores. Para o presente estudo foram utilizadas as medidas de ajuste CFI, pois tem a vantagem de evitar a subestimação do ajuste observado no NFI quando a amostra é pequena, TLI, similar ao CFI, e RMSEA, pois representa mais adequadamente quão bem um modelo se ajusta à população, não apenas à amostra utilizada para a estimação (THOMPSON, 2004).

\section{Análise dos Resultados}

A pesquisa foi operacionalizada através da utilização de um questionário estruturado, aplicado entre os meses de maio e agosto de 2016 junto à gestores de Tecnologia da Informação. Cada gestor poderia responder ao questionário duas vezes, uma vez para uma situação de insatisfação e posterior recuperação do serviço, mas que a relação com o fornecedor continuou e outra vez para uma situação de insatisfação e posterior recuperação de serviços, mas que o cliente escolheu trocar de fornecedor. Ao todo 134 empresas participaram do estudo, com um total de 171 questionários respondidos.

Em relação ao tamanho das empresas, obteve-se uma grande variação. Os valores vão desde empresas com apenas 5 funcionários, até grandes empresas com mais de 22.000 funcionários. Na pesquisa puderam ser identificadas relações comerciais que duram/duraram 6 meses, até relações de longa duração, que duram/duraram 24 anos. Entre as empresas que participaram do estudo, o maior percentual, de 52,24\%, foi das empresas do setor de serviços em seguida as empresas do setor de comércio com $28,36 \%$, instituições governamentais com 13,43\% e indústrias com 5,97\%. 
http://www.revista.ufpe.br/gestaoorg

Devido ao fato de a amostra ter sido coletada por conveniência, grande parte das empresas participantes do estudo encontram-se no estado de Pernambuco, chegando a um percentual de $89,55 \%$ do total de empresas pesquisadas.

\subsection{Confiabilidade do Instrumento}

De acordo com Da Hora, Monteiro e Arica (2010) o coeficiente alfa de Cronbach tem grande utilização e aceitação no meio acadêmico como ferramenta para estimação da confiabilidade do questionário. Hair Jr. et al. (2009) afirmam que valores de alfa de Cronbach acima de 0,6 são considerados satisfatórios. Os valores obtidos para o alfa de Cronbach das variáveis pesquisadas estão disponíveis na Tabela 2.

Tabela 2: Alfa de Cronbach Calculado para as Escalas Utilizadas no Estudo.

\begin{tabular}{|l|c|}
\hline Construto & Alfa de Cronbach \\
\hline Confiança & 0,938 \\
\hline Dependência do Fornecedor & 0,807 \\
\hline Severidade da Falha & 0,869 \\
\hline Comprometimento Calculativo & 0,702 \\
\hline
\end{tabular}

Fonte: Coleta de dados (2016)

Apesar de as escalas utilizadas no estudo foram obtidas a partir de instrumentos já aplicados e validados em estudos anteriores foi realizado um estudo de análise fatorial confirmatória, conforme sugerido por Hair Jr. et al. (2009). Todos os itens dos questionários apresentaram carga fatorial superior a 0,7.

\subsection{Análise do modelo estrutural}

O primeiro passo na análise do modelo estrutural consiste na avaliação de problemas relacionados com a colinearidade. Para avaliação da multicolinearidade utiliza-se o VIF (variance inflation factor). Valores de VIF acima de 5 são considerados indesejáveis (HAIR et al., 2012). Para o modelo do estudo os valores de VIF ficaram entre 1,032 e 1,293.

As relações entre confiança e comprometimento calculativo, e dependência e comprometimento calculativo se mostraram significativas. Apenas a relação entre severidade da falha e comprometimento calculativo não obteve suporte estatístico. A variância explicada a partir do modelo para a variável comprometimento calculativo foi de $49,7 \%$.

Para as relações entre a confiança e a dependência, e a confiança e a severidade da falha, obtiveram suporte estatístico. A variância explicada a partir do modelo para a variável confiança foi de $30,7 \%$. Os valores obtidos na regressão podem ser observados na Tabela 3.

Tabela 3: Resultado das Regressões.

\begin{tabular}{|l|l|l|l|l|}
\hline Relações & Estimate & S.E & $\boldsymbol{P}$ & $\mathbf{R}^{\mathbf{2}}$ \\
\hline Confiança <--- Severidade & $-0,327$ & 0,076 & 0,000 & \multirow{2}{*}{$30,7 \%$} \\
\cline { 1 - 4 } Confiança <--- Dependência & 0,437 & 0,083 & 0,000 & \\
\hline Comprometimento Calculativo <--- Dependência & 0,262 & 0,092 & 0,004 & \multirow{2}{*}{$49,7 \%$} \\
\cline { 1 - 3 } Comprometimento Calculativo <--- Confiança & 0,474 & 0,098 & 0.000 & \\
\cline { 1 - 3 } Comprometimento Calculativo <--- Severidade & $-0,086$ & 0,079 & 0,278 & \\
\cline { 1 - 3 }
\end{tabular}

Fonte: Coleta de dados (2016)

O presente estudo utilizou as medidas de ajuste CFI, onde valores acima de 0,90 são considerados aceitáveis e os valores encontrados no estudo foram de $\mathbf{0 , 9 4 0}$, TLI, o qual aceita valores acima de 0,90 , sendo o valor de $\mathbf{0 , 9 2 7}$ encontrado nos estudos, e RMSEA, que permite valores de até 0,08 , o qual obteve $\mathbf{0 , 0 7 6}$ no presente estudo (THOMPSON, 2004). 
A partir do resultado obtido nas regressões é possível que sejam analisadas as hipóteses apresentadas no presente estudo. Para as relações entre confiança e severidade, na qual o modelo teórico propôs uma relação negativa, e confiança e dependência, na qual o modelo teórico propôs uma relação positiva, houve suporte estatístico, suportando as hipóteses $\mathrm{H} 2$ e H4.

Para a relação entre comprometimento calculativo e dependência, a qual o modelo teórico propôs uma relação positiva, houve suporte estatístico, suportando a hipótese H3. A relação entre comprometimento calculativo e confiança, também obteve suporte estatístico, suportando a hipótese H1. Apenas a relação entre comprometimento calculativo e severidade da falha, a qual o modelo teórico propôs ser negativa, não pode ser suportada estatisticamente, refutando a hipótese H5. O modelo resultante e os valores obtidos nas regressões podem ser observados na Figura 2.

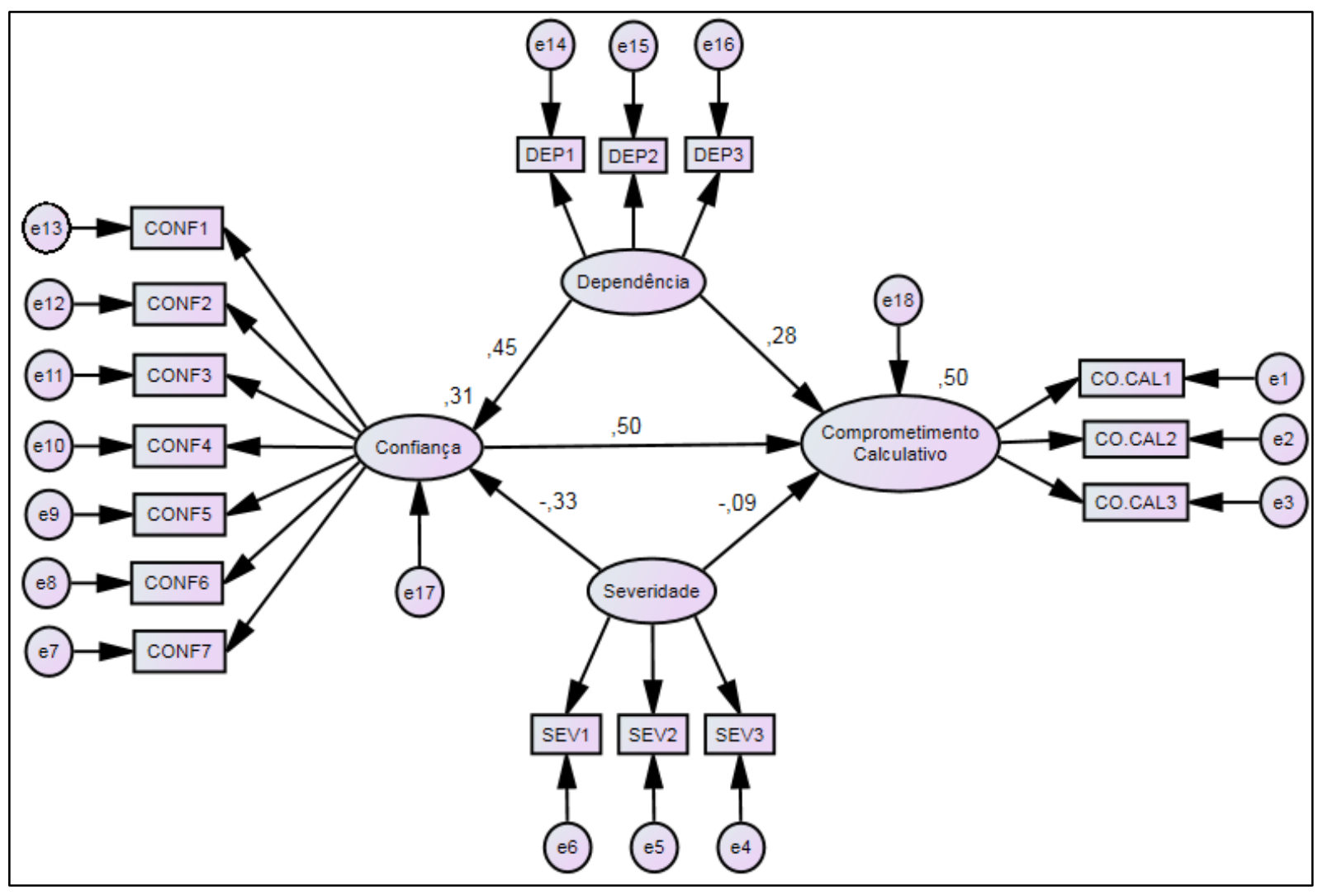

Figura 2: Modelo do Resultado da MEE

Fonte: Elaborado pelos autores

\section{Conclusões}

Utilizar estratégias de recuperação que resultem na retenção de clientes é um fator fundamental para a sobrevivência. Conhecer as saídas dos momentos de falha, como aumento/redução do comprometimento e da confiança (TAX et al., 1998), e a influência de ações anteriores à falha, como dependência do fornecedor (YANAMANDRAM; WHITE, 2010) e como estes se relacionam podem representar a diferença entre manter ou perder o cliente.

Em relações de longo prazo, que podem sofrer de eventuais flutuações na qualidade do serviço, existirão momentos de insatisfação do cliente em relação ao serviço prestado pelo fornecedor, a recuperação da falha pode gerar maiores níveis de Confiança e Comprometimento, conforme proposto por Tax et al. (1998).

Vários são os estudos que analisam o papel da Severidade da Falha em vários aspectos do marketing de serviços e da retenção de clientes. Entre eles é possível citar os estudos de Weun et al. (2004), McQuilken e Robertson (2011) e Wang e Chang (2013). Em todos os estudos citados o ambiente pesquisado estava inserido em contextos B2C. Uma contribuição importante do presente estudo é a análise do impacto da severidade em contextos B2B.

Uma importante noção que pode ser levantada a partir dos resultados é que, como a confiança é um construto antecedente do comprometimento (CHANG et al., 2012; GOUNARIS, 2005; WANG; CHANG, 2013), a relação da dependência do fornecedor como antecedente da confiança (BELDAD et al., 2010), pode levar a níveis mais elevados de certas dimensões do comprometimento, como o comprometimento calculativo. 
http://www.revista.ufpe.br/gestaoorg

A TI é um setor no qual grande parte das operações é realizada a partir de provedores de serviços externo, e que a confiança entre as partes é um requerimento para que ocorra a efetiva operação. No presente estudo, realizado em um contexto de prestação de serviços B2B, em uma atividade com grande grau de contratação de serviços terceirizados, a relação entre a dependência e a confiança pode ser verificada, corroborando com o proposto nos estudos de Beldad et al. (2010).

Proporcionar serviços de alta qualidade, garantindo a satisfação do cliente, ainda é a receita para a retenção e o sucesso das empresas. Mas é preciso estar preparado para os momentos de falha e saber que, mesmo quando o erro ocorre, é possível manter altos níveis de retenção, garantindo a longevidade do negócio.

\subsection{Implicações Gerenciais e Acadêmicas}

A presente pesquisa teve o objetivo de analisar as relações entre variáveis antecedentes do comprometimento calculativo em contextos de relação B2B após a ocorrência de falhas de serviço. O referido contexto é algo pouco estudado e sua avaliação das relações entre as diferentes variáveis permite maior entendimento sobre o tema, permitindo o avanço científico na área de Marketing.

Um importante achado do estudo, com implicações acadêmicas e gerenciais, foram os papeis das variáveis confiança e severidade da falha Henning-Thurau e Klee (1997) argumentam que o marketing de relacionamento, o qual é composto por confiança, comprometimento e qualidade percebida, é parte central e determinante para a retenção de clientes, e no modelo resultante do presente estudo a variável confiança representou um papel de grande importância.

Para gerentes de serviços de TI, é fundamental adotar procedimentos que permitam gerar altos níveis de confiança, como também ferramentas que evitem que falhas de serviços sejam percebidas como graves. Como as falhas de serviços são inevitáveis, a utilização de protocolos para mapeá-las e diminuir seu impacto para o negócio do cliente pode ser a diferença entre reter ou perder o cliente.

O “ir além”, proposto por Hollmann et al. (2015), está em consonância com os achados do presente estudo. Perceber que as falhas vão ocorrer e que é preciso trata-la corretamente, gerar relacionamentos próximos de confiança e comprometimento e perceber o que é, de fato, importante para o cliente, não só organizacionalmente, mas, também, no nível pessoal.

\section{Referências}

ALBERTIN, A.L.; ALBERTIN, R.M. Dimensões do uso de tecnologia da informação: um instrumento de diagnóstico e análise. Revista de Administração Pública, v. 46, n. 1, p. 125-151, 2012.

ANG L; BUTTLE F. Customer retention management process: A quantitative study". Eur. J. Mark., 40 (1): 83 99, 2006.

BEATSON, A; LEE, N; COOTE, LV. Self-Service Technology and the Service Encounter. Service Industries Journal. 27, 1, 75-89, Jan. 2007. ISSN: 02642069.

BELDAD, Ardion; DE JONG, Menno; STEEHOUDER, Michaël. How shall I trust the faceless and the intangible? A literature review on the antecedents of online trust. Computers in Human Behavior, v. 26, n. 5, p. 857-869, 2010.

BERNSTORFF, V. H.; CUNHA, J. C. O que as Organizações Buscam e Alcançam com a Terceirização em Tecnologia da Informação (TI). IN: XXIII Encontro da ANPAD, v. 23, 2000.

BERRY, Leonard L.; PARASURAMAN, A. Marketing service. Competing through Quality, New York, 1991.

BITNER, M.J. Services and technology: opportunities and paradoxes, Managing Service Quality, 11(6), pp.3759, 2001.

BITNER, M.J.; BOOMS, B.H.; TETREAULT, M.S. The service encounter: Diagnosing Favorable and Unfavorable Incidents. Journal of Marketing. 54, 1,71-84, Jan. 1990. ISSN: 00222429.

CHANG, S. H.; WANG, K. Y.; CHIH, W. H.; TSAI, W. H. Building customer commitment in business-tobusiness markets. Industrial marketing management, v. 41, n. 6, p. 940-950, 2012.

CHURCHILL JR, G.A.; SURPRENANT, C. An investigation into the determinants of customer satisfaction. Journal of marketing research, p. 491-504, 1982.

CURRAN, J.M., MEUTER, M.L. e SURPRENANT, C.F. Intentions to use self-service technologies: a confluence of multiple attitudes, Journal of Service Research, 5(3), pp.209-24, 2003.

DA HORA, H. R. M.; MONTEIRO, G. T. R.; ARICA, J. Confiabilidade em questionários para qualidade: um estudo com o Coeficiente Alfa de Cronbach. Produto \& Produção, v. 11, n. 2, p. 85-103, 2010. 
DE ANDRADE, C. H. M.; REZENDE, S. F. L.; SALVATO, M. A.; BERNARDES, P. A Relação entre Confiança e Custos de Transação em Relacionamentos Interorganizacionais / The Relation between Trust and Transaction Costs in Interorganizational Relationships. Revista de Administração Contemporânea, v. 15, n. 4, p. 608, 2011.

DONEY, P.M.; BARRY, J.M.; ABRATT, R. Trust determinants and outcomes in global B2B services, Vol. 41 Iss: 9/10, pp.1096-1116, 2007.

DONEY, P. M.; CANNON, J. P. An examination of the nature of trust in buyer-seller relationships. Journal of Marketing, v. 61, n. 2, p. 35-51, 1997.

GAO, T.; SIRGY, M. J.; BIRD, M. M. Reducing buyer decision-making uncertainty in organizational purchasing: can supplier trust, commitment, and dependence help?. Journal of Business Research, v. 58, n. 4, p. 397-405, 2005.

GANESAN, S. Determinants of long-term orientation in buyer-seller relationships. the Journal of Marketing, $p$. $1-19,1994$.

GEYSKENS, I.; STEENKAMP, J.B.E.; SCHEER, L.K.; KUMAR, N. The effects of trust and interdependence on relationship commitment: A trans-Atlantic study. International Journal of research in marketing, v. 13, n. 4, p. 303-317, 1996.

GILLILAND, D. I.; BELLO, D. C. Two sides to attitudinal commitment: The effect of calculative and loyalty commitment on enforcement mechanisms in distribution channels. Journal of the Academy of Marketing Science, v. 30, n. 1, p. 24-43, 2002.

GOUNARIS, S.P. Trust and commitment influences on customer retention: insights from business-to-business services. Journal of Business research, v. 58, n. 2, p. 126-140, 2005.

GUSTAFSSON, A.; JOHNSON, M. D.; ROOS, I. The effects of customer satisfaction, relationship commitment dimensions, and triggers on customer retention. Journal of marketing, v. 69, n. 4, p. 210-218, 2005.

HAIR, Joe F. et al. An assessment of the use of partial least squares structural equation modeling in marketing research. Journal of the academy of marketing science, v. 40, n. 3, p. 414-433, 2012.

HAIR Jr., J. F.; BLACK,W. C.; BABIN, B. J.; ANDERSON, R. E.; TATHAM, R. L. Análise multivariada de dados. 6. ed. Porto Alegre: Bookman, 2009.

HEIDE, J.B.; JOHN, G. Do norms matter in marketing relationships?. The Journal of Marketing, p. 32-44, 1992.

HENNIG-THURAU, T.; KLEE; A. The impact of customer satisfaction and relationship quality on customer retention: a critical reassessment and model development. Psychology \& Marketing, v. 14, n. 8, p. 737-764, 1997.

HOLLMANN, T; JARVIS, C; BITNER, M. Reaching the breaking point: a dynamic process theory of businessto-business customer defection. Journal of the Academy of Marketing Science. 43, 2, 257-278, Mar. 2015. ISSN: 00920703.

HUME, M.; MCCOLL-KENNEDY, J.R. Episodic, extended and continuous service encounters: a theoretical framework. In: Conference Proceedings ANZMAC Conference, Sydney. 1999.

JIANG; Z.; HENNEBERG, S. C.; NAUDÉ, P. The importance of trust vis-à-vis reliance in business relationships: some international findings. International Marketing Review, v. 28, n. 4, p. 318-339, 2011.

JONES, M. A.; REYNOLDS, K. E.; MOTHERSBAUGH, D. L.; BEATTY, S. E. The positive and negative effects of switching costs on relational outcomes. Journal of Service Research, v. 9, n. 4, p. 335-355, 2007.

KUMAR, N.; STERN, L. W.; ANDERSON, J. C. Conducting interorganizational research using key informants. Academy of management journal, v. 36, n. 6, p. 1633-1651, 1993.

LOVELOCK, C. Product Plus: How Product + Service = Competitive Advantage, McGraw-Hill, Inc., New York, NY, 1994.

LOVELOCK, C.H.; WIRTZ, J. Marketing de serviços: pessoas, tecnologia e resultados. Pearson P. Hall, 2011.

MALHOTRA, N. K.; BIRKS, D.; WILLS, P. Marketing research: applied approach. 4th edition. New York: Pearson, 2012.

MCCOLLOUGH, Michael A. The recovery paradox: The effect of recovery performance and service failure severity on post-recovery customer satisfaction. Academy of Marketing Studies Journal, v. 13, n. 1, p. 89, 2009.

MCFARLAN, W.E. Information Technology Changes The Way You Compete. Harvard Business Review, v.62, n.3, p.98-103, May/June 1984.

MCQUILKEN, Lisa. The influence of failure severity and employee effort on service recovery in a service guarantee context. Australasian Marketing Journal (AMJ), v. 18, n. 4, p. 214-221, 2010. 
http://www.revista.ufpe.br/gestaoorg

MCQUILKEN, Lisa; ROBERTSON, Nichola. The influence of guarantees, active requests to voice and failure severity on customer complaint behavior. International Journal of Hospitality Management, v. 30, n. 4, p. 953 962, 2011.

MORGAN, R.M.; HUNT, S.D. The commitment-trust theory of relationship marketing. Journal of Marketing, v. 58, n. 3, p. 20-38, 1994.

MOUZAS, S.; HENNEBERG, S.; NAUDÉ, P. Trust and reliance in business relationships. European Journal of Marketing, v. 41, n, 9/10, p. 1016-1032, 2007.

NESPOLO, D. Relação entre valor percebido, reputação, confiança e custos de troca como determinantes da retenção de clientes no contexto de serviços de telefonia móvel. 2014.

OLIVER, R.L. A Cognitive Model of the Antecedents and Consequences of Satisfaction Decisions. Journal of Marketing Research (JMR). 17, 4, 460-469, Nov. 1980. ISSN: 00222437.

OLIVER, R.L.; DESARBO, W.S. Response determinants in satisfaction judgments. Journal of consumer research, p. 495-507, 1988.

PALMATIER, R.W.; GOPALAKRISHNA, S.; HOUSTON, M. B. Returns on business-to-business relationship marketing investments: Strategies for leveraging profits. Marketing Science, v. 25, n. 5, p. 477-493, 2006.

PIZZUTTI DOS SANTOS, Cristiane; BASSO, Kenny. Do ongoing relationships buffer the effects of service recovery on customers' trust and loyalty?. International Journal of Bank Marketing, v. 30, n. 3, p. 168-192, 2012.

PORTER, Michael E.; MILLAR, Victor E. How information gives you competitive advantage. 1985.

RUSBULT, Caryl E. Commitment and satisfaction in romantic associations: A test of the investment model. Journal of experimental social psychology, v. 16, n. 2, p. 172-186, 1980.

SHEPPARD. B. H.; SHERMAN, D. M. The grammars of trust: a model and general implications. Academy of Management Review, v. 23, n. 3, p. 422-437, 1998.

SILVA, H. C. C.; ARAÚJO, M. A. V. Determinantes de satisfação e insatisfação em serviços de suporte à tecnologia da informação em ambientes B2B. Anais - SIMPEP, 2015.

SIQUEIRA, M.M.M. Comprometimento organizacional afetivo, calculativo e normativo: evidências acerca da validade discriminante de três medidas brasileiras. Encontro Anual da ANPAD, v. 25, p. 23-40, 2001.

TANG, Andrew C. Justice-Based Normative Recovery Expectations in Enterprise Information Technology Services: The Effect of Failure Severity and Criticality on Disconfirmation and Satisfaction. 2014.

TAX, S.S.; BROWN, S.W.; CHANDRASHEKARAN, M. Customer Evaluations of Service Complaint Experiences: Implications for Relationship Marketing. Journal of Marketing. 62, 2, 60-76, Apr. 1998. ISSN: 00222429.

THOMPSON, Bruce. Exploratory and confirmatory factor analysis: Understanding concepts and applications. American Psychological Association, 2004.

TSE, D.K.; WILTON, P.C. Models of consumer satisfaction formation: An extension. Journal of marketing research, p. 204-212, 1988.

VENETIS, K.A.; GHAURI, P.N. Service quality and customer retention: building long-term relationships. European Journal of marketing, v. 38, n. 11/12, p. 1577-1598, 2004.

WANG, E. S.; CHANG, S. Creating Positive Word-of-Mouth Promotion Through Service Recovery Strategies. Services Marketing Quarterly, v. 34, n. 2, p. 103-114, 2013.

WANG, Y. S.; WU, S. C.; LIN, H. H.; WANG, Y. Y. The relationship of service failure severity, service recovery justice and perceived switching costs with customer loyalty in the context of e-tailing. International Journal of Information Management, v. 31, n. 4, p. 350-359, 2011.

WETZELS, M.; DE RUYTER, K.; VAN BIRGELEN, M. Marketing service relationships: the role of commitment. Journal of business \& industrial marketing, v. 13, n. 4/5, p. 406-423, 1998.

WEUN, S.; BEATTY, S. E.; JONES, M.A. The impact of service failure severity on service recovery evaluations and post-recovery relationships. Journal of Services Marketing, v. 18, n. 2, p. 133-146, 2004.

WHITE, L.; YANAMANDRAM, V. A model of customer retention of dissatisfied business services customers. Managing Service Quality: An International Journal, v. 17, n. 3, p. 298-316, 2007.

YANAMANDRAM, V.; WHITE, L. An empirical analysis of the retention of dissatisfied business services customers using structural equation modelling. Australasian Marketing Journal (AMJ), v. 18, n. 4, p. 222-232, 2010. 